\title{
On Size and Shape of the Average Meson Fields in the Semibosonized Nambu \& Jona-Lasinio Model
}

\author{
R. WÜnsch ${ }^{1}$, K. GOekE ${ }^{2}$, Th. Meissner ${ }^{3}$ \\ ${ }^{1}$ Institut für Kern- und Hadronenphysik, Forschungszentrum Rossendorf e.V., \\ Postfach 5101 19, 01314 Dresden, Germany \\ ${ }^{2}$ Institut für Theoretische Physik II, Ruhr-Universität Bochum, \\ Postfach 1021 48, 44780 Bochum, Germany \\ ${ }^{3}$ Institute for Nuclear Theory, University of Washington, HN-12 Seattle, \\ WA 98195 , USA
}

\begin{abstract}
We consider a two-flavor Nambu \& Jona-Lasinio model in Hartree approximation involving scalar-isoscalar and pseudoscalar-isovector quark-quark interactions. Average meson fields are defined by minimizing the effective Euklidean action. The fermionic part of the action, which contains the full Dirac sea, is regularized within Schwinger's proper-time scheme. The meson fields are restricted to the chiral circle and to hedgehog configurations. The only parameter of the model is the constituent quark mass $M$ which simultaneously controls the regularization.

We evaluate meson and quark fields self-consistently in dependence on the constituent quark mass. It is shown that the self-consistent fields do practically not depend on the constituent quark mass. This allows us to define a properly parameterized reference field which for physically relevant constituent masses can be used as a good approximation to the exactly calculated one. The reference field is chosen to have correct behaviour for small and large radii.

To test the agreement between self-consistent and reference fields we calculate several observables like nucleon energy, mean square radius, axial-vector constant and deltanucleon mass splitting in dependence on the constituent quark mass. The agreement is found to be very well.
\end{abstract}




\section{Introduction}

The model of Nambu \& Jona-Lasinio (NJL) [1] has been used quite successfully as effective chiral theory for low and medium energy hadronic phenomena. First it has been applied to vacuum and meson properties as well as medium effects (for reviews c. f. [2, 3, 田). Later on it turned out that also baryonic systems (nucleons and hyperons) can be described within this model (for a review c. f. [5]). Starting from a semi-bosonized version [6, 7] with scalar-isoscalar and pseudoscalar-isovector interaction and treating the meson fields classically various authors have shown that for constituent quark masses $M \gtrsim 350 \mathrm{MeV}$ it is possible to get self-consistent solitonic solutions with baryon number $\mathrm{B}=1$ consisting of 3 valence quarks in addition to the polarized Dirac sea [8]-[17]. Because of the nonrenormalizability of the Nambu \& Jona-Lasinio model the sea contribution diverges and has to be regularized. The parameters of the model can be fixed to the physics of the meson and vacuum sector, mainly to the weak pion decay constant $f_{\pi}$ and the pion mass $m_{\pi}$. In doing so only one free parameter remains open for the baryonic sector for which we take the constituent quark mass $M$.

The self-consistent determination of the meson fields is a time-consuming numerical procedure. Changing the parameters of the model or the regularization scheme the procedure has to be repeated again. So it might be helpful to look for an analytic parametrization of the selfconsistent profile function $\Theta(r)$ of the solitonic solution which approximates the exact $\Theta(r)$ as well as possible. Within the restrictions to hedgehog configurations [18 and to the chiral circle the meson fields are uniquely described by the profile function $\Theta(r)$. In the course of our calculations we noticed to a very large extent an independence of this profile function on the constituent quark mass $M$. It is the aim of this paper to investigate this dependence quantitatively and to look for a general function which may approximate the profile function, if possible independently of $M$.

In section 2 we review the main ideas of the semi-bosonized and regularized Nambu \& Jona-Lasinio model for two flavors and introduce observables characterizing the quark and meson configuration. The dependendence of the self-consistent meson profiles $\Theta(r)$ on the constituent quark mass $M$ is investigated in sect. 3 within a wide range $(350 \mathrm{MeV} \leq M \leq$ $1000 \mathrm{MeV})$. Here we compare the self-consistent profile with a reference profile $\Theta^{R e f}(R ; r)$ given by a simple arithmetic expression obtained from an asymptotic expansion of the equation of motion at $r \rightarrow 0$ and $r \rightarrow \infty$. In sect. 4 we evaluate several observables like nucleon mass, mean-square radius, axial-vector coupling constant and delta-nucleon mass splitting using both the self-consistently determined profiles and the standard profiles. The comparison of both values tests and illustrates the quality of the reference profile.

\section{The regularized and bosonized Nambu \& Jona- Lasinio model and its observables}

The details of the following section can be found in ref. 15, 16, 17, 21]. Here we shortly review these parts of the formalism which make this paper self-contained.

We consider a two-flavor NJL lagrangian

$$
\mathcal{L}_{N J L}(\bar{q} q)=\bar{q}[i \not \partial-m] q+\frac{G}{2}\left[(\bar{q} q)^{2}+\left(\bar{q} i \gamma_{5} \hat{\boldsymbol{\tau}} q\right)^{2}\right]
$$

for the quark fields $q(x)$ ( $u$ and $d$ quarks of $N_{c}=3$ colours). Here $G$ is the coupling constant, $\hat{\boldsymbol{\tau}}$ the vector of Pauli-matrices and $m$ the average current mass of the light 
quarks $m=\left(m_{u}+m_{d}\right) / 2$. The theory with only quark degrees of freedom is converted into an effective quark-meson theory by means of standard path-integral bosonization [6, 0, 19]. The mesonic fields are restricted to hedgehog configurations and to the ciral circle. The resulting semi-bosonized theory is described by an effective (Euklidean) action, whose dynamical quantity is the profile function $\Theta(r)$ [10, 11]

$$
\mathcal{A}_{\text {eff }}[\Theta]=\mathcal{A}^{q}[\Theta]+\mathcal{A}^{m}[\Theta]
$$

The $\mathcal{A}_{\text {eff }}[\Theta]$ consists of a quark part

$$
\mathcal{A}^{q}[\Theta]=-\operatorname{Sp} \ln \left[\beta\left(\frac{\partial}{\partial \tau}+h-\mu\right)\right]+S p \ln \left[\beta\left(\frac{\partial}{\partial \tau}+h_{V}\right)\right]
$$

with the quark hamiltonian

$$
h=\boldsymbol{\alpha} \cdot \boldsymbol{p}+g f_{\pi} \beta\left[\cos \Theta(r)+i \sin \Theta(r) \gamma_{5} \hat{\boldsymbol{\tau}} \cdot \hat{\boldsymbol{r}}\right],
$$

and of a meson part

$$
\mathcal{A}^{m}[\Theta]=\mathcal{T} m f_{\pi} \frac{\lambda^{2}}{g} 4 \pi \int r^{2} d r[1-\cos \Theta(r)],
$$

where $\mathcal{T}$ is the total Euklidean time interval. The quark-meson coupling strength $g$ in the hamiltonian (4) and the parameter $\lambda$ in the mesonic action (5) are related to the interaction strength $G$ in the NJL lagrangian (11) via $G=g^{2} / \lambda^{2}$. Since we are interested in stationary field configurations we have assumed the meson fields to be timeindependent. Hence the eigenvalues $\varepsilon_{\alpha}$ of the hamiltonian (雨) are real. The vaccum state (with broken chiral symmetry) has $\Theta(r) \equiv 0$ and and the eigenvalues and eigenfunctions of corresponding hamiltonian are denoted by $\varepsilon_{\alpha}^{V}$ and $\Phi_{\alpha}^{V}(\boldsymbol{r})$, respectively. The symbol $S p$ indicates functional and matrix (spin, isospin, colour) trace

$$
S p \mathcal{O} \equiv N_{c} t r_{\gamma} t r_{\tau} \int d^{4} x_{E}\left\langle x_{E}|\mathcal{O}| x_{E}\right\rangle
$$

with the Euklidean space-time vector $x_{E}^{\mu}=(\tau, \boldsymbol{r})$ and its volume element $d^{4} x_{E}$. The effective quark action (3) can be divided into a valence contribution

$$
\mathcal{A}_{v a l}^{q}[\Theta] \equiv \mathcal{A}^{q}[\Theta](\mu)-\mathcal{A}^{q}[\Theta](\mu=0)=-\mathcal{T} \frac{N_{c}}{2} \sum_{\alpha}\left[\left|\varepsilon_{\alpha}-\mu\right|-\left|\varepsilon_{\alpha}\right|\right]
$$

and a sea contribution

$$
\mathcal{A}_{\text {sea }}^{q}[\Theta] \equiv \mathcal{A}^{q}[\Theta](\mu=0)=-\mathcal{T} \frac{N_{c}}{2} \sum_{\alpha}\left[\left|\varepsilon_{\alpha}\right|-\left|\varepsilon_{\alpha}^{V}\right|\right] .
$$

The valence contribution (7) depends on the chemical potential $\mu$ which will be adjusted such that the resulting soliton has baryon number one. This can be achieved by a chemical potential $\mu>0$ which is slightly larger than the energy $\varepsilon_{v a l}$ of the lowest quark level with positive energy (valence level). However, the meson field may be so strong that the valence level is bent down to the Dirac sea $\left(\varepsilon_{v a l}<0\right)$. In this case the Dirac sea has one level more than in vacuum state and therefore carries baryon number one. Describing a baryon with baryon number one we have to choose $\mu=0$, in this case, making the Fermi sea $\left(0 \leq \varepsilon_{\alpha} \leq \mu\right)$ empty. 
The sea contribution (8) diverges and is regularized. Applying Schwinger's propertime scheme $\left.\ln \mathcal{O} \longrightarrow \ln \mathcal{O}\right|_{R e g}=-\int_{1 / \Lambda^{2}}^{\infty} \frac{d s}{s} e^{-s \mathcal{O}}$ [20], where $\Lambda$ is the regularization parameter, we get a regularized sea contribution

$$
\mathcal{A}_{\text {sea }}^{q, R e g}=-\mathcal{T} \frac{N_{c}}{2} \sum_{\alpha}\left[R_{E}\left(\varepsilon_{\alpha}, \Lambda\right)\left|\varepsilon_{\alpha}\right|-R_{E}\left(\varepsilon_{\alpha}^{V}, \Lambda\right)\left|\varepsilon_{\alpha}^{V}\right|\right] .
$$

Here the regularization function is given by

$$
R_{E}(\varepsilon, \Lambda)=-\frac{1}{\sqrt{4 \pi}|\varepsilon|} \int_{1 / \Lambda^{2}}^{\infty} d t t^{-3 / 2} e^{-\varepsilon^{2} t}=-\frac{1}{\sqrt{4 \pi}} \Gamma\left(-\frac{1}{2}, \frac{\varepsilon^{2}}{\Lambda^{2}}\right)
$$

with the incomplete Gammafunction $\Gamma(x, a)$. The total regularized effective action reads

$$
\mathcal{A}_{\text {eff }}^{\operatorname{Reg}}[\Theta]=\mathcal{A}_{\text {val }}^{q}[\Theta]+\mathcal{A}_{\text {sea }}^{q, \operatorname{Reg}}[\Theta]+\mathcal{A}^{m}[\Theta] .
$$

The profile function $\Theta(r)$ is determined by

$$
\frac{\delta \mathcal{A}_{\text {eff }}^{\operatorname{Reg}}[\Theta]}{\delta \Theta(r)}=0
$$

yielding the equation of motion

$$
\Theta(r)=\arctan \frac{\bar{P}(r)}{\bar{S}(r)}
$$

with

$$
\bar{S}(r)=\frac{m}{g}-\frac{g}{\lambda^{2}}\left[N_{c} \theta\left(\varepsilon_{v a l}\right) \bar{S}_{v a l}(r)-\frac{N_{c}}{2} \sum_{\alpha} R_{m}\left(\varepsilon_{\alpha}, \Lambda\right) \bar{S}_{\alpha}(r)\right]
$$

and

$$
\begin{gathered}
\bar{S}_{\alpha}(r)=\frac{1}{4 \pi} \int d \hat{\boldsymbol{r}} \bar{\Phi}_{\alpha}(\boldsymbol{r}) \Phi_{\alpha}(\boldsymbol{r}) \\
\bar{P}(r)=-\frac{g}{\lambda^{2}}\left[N_{c} \theta\left(\varepsilon_{v a l}\right) \bar{P}_{v a l}(r)-\frac{N_{c}}{2} \sum_{\alpha} R_{m}\left(\varepsilon_{\alpha}, \Lambda\right) \bar{P}_{\alpha}(r)\right]
\end{gathered}
$$

and

$$
\bar{P}_{\alpha}(r)=\frac{1}{4 \pi} \int d \hat{\boldsymbol{r}} \bar{\Phi}_{\alpha}(\boldsymbol{r}) i \gamma_{5} \hat{\boldsymbol{r}} \cdot \hat{\boldsymbol{\tau}} \Phi_{\alpha}(\boldsymbol{r}) .
$$

The valence contributions $\bar{S}_{v a l}(r)$ and $\bar{P}_{v a l}(r)$ are obtained for the valence level $\alpha=v a l$. They vanish if the valence level dips into the Dirac sea $\left(\varepsilon_{v a l}<0\right)$. The $\Phi_{\alpha}(\boldsymbol{r})$ are the eigenfunctions of $h$. The sea contributions have been regularized within the proper-time scheme with a regularization function

$$
R_{m}(\varepsilon, \Lambda)=\frac{\varepsilon}{\sqrt{\pi}} \int_{1 / \Lambda^{2}}^{\infty} d t t^{-1 / 2} e^{-\varepsilon^{2} t}=\frac{\operatorname{sign}(\varepsilon)}{\sqrt{\pi}} \Gamma\left(\frac{1}{2}, \frac{\varepsilon^{2}}{\Lambda^{2}}\right) .
$$

The parameters of the model will be fixed by adjusting the vaccuum and meson sectors as described in detail in ref. [16]. The only remaining free parameter is the constituent quark mass 


$$
M=g f_{\pi}
$$

which will be varied within reasonable limits.

In this paper we consider the (isoscalar) baryon density

$$
\rho(\boldsymbol{r})=\frac{1}{N_{c}}\left\langle: q^{\dagger}(\boldsymbol{r}) q(\boldsymbol{r}):\right\rangle=\rho(\boldsymbol{r})_{v a l}+\rho(\boldsymbol{r})_{\text {sea }}
$$

with

$$
\rho(\boldsymbol{r})_{v a l}=N_{c} \theta\left(\varepsilon_{v a l}\right) \Phi_{v a l}^{\dagger}(\boldsymbol{r}) \Phi_{v a l}(\boldsymbol{r})
$$

and

$$
\rho(\boldsymbol{r})_{\text {sea }}=-\frac{1}{2} \sum_{\alpha}\left[\operatorname{sign}\left(\varepsilon_{\alpha}\right) \Phi_{\alpha}^{\dagger}(\boldsymbol{r}) \Phi_{\alpha}(\boldsymbol{r})-\operatorname{sign}\left(\varepsilon_{\alpha}^{V}\right) \Phi_{\alpha}^{V \dagger}(\boldsymbol{r}) \Phi_{\alpha}^{V}(\boldsymbol{r})\right] .
$$

The colon in the matix element of eq. (20) indicates subtraction of the corresponding value calculated in the vacuum state. Like for any other observable the valence contribution (21) vanishes if the Fermi sea is empty. The Dirac sea contribution (22) has not been regularized because it is finite and fulfills the exact normalization condition (baryon number zero/one) only in the unregularized case [16]. The size of the density distribution is characterized by the mean square baryon radius

$$
\bar{R} \equiv \sqrt{\left\langle R^{2}\right\rangle}=\left[\int d^{3} r r^{2} \rho(\boldsymbol{r})\right]^{1 / 2}
$$

Another quantity characterizing a quark configuration is the axial density

$$
A_{o}(\boldsymbol{r})=\left\langle: q^{\dagger}(\boldsymbol{r}) \frac{\sigma_{o} \tau_{o}}{2} q(\boldsymbol{r}):\right\rangle
$$

It consists of a valence contribution

$$
A_{o}(\boldsymbol{r})_{v a l}=N_{c} \theta\left(\varepsilon_{v a l}\right) \Phi_{v a l}^{\dagger}(\boldsymbol{r}) \frac{\sigma_{o} \tau_{o}}{2} \Phi_{v a l}(\boldsymbol{r})
$$

and of a sea contribution

$$
A_{o}(\boldsymbol{r})_{\text {sea }}=-\frac{N_{c}}{2} \sum_{\alpha}\left[R_{m}\left(\varepsilon_{\alpha}, \Lambda\right) \Phi_{\alpha}^{\dagger}(\boldsymbol{r}) \frac{\sigma_{o} \tau_{o}}{2} \Phi_{\alpha}(\boldsymbol{r})-R_{m}\left(\varepsilon_{\alpha}^{V}, \Lambda\right) \Phi_{\alpha}^{V \dagger}(\boldsymbol{r}) \frac{\sigma_{o} \tau_{o}}{2} \Phi_{\alpha}^{V}(\boldsymbol{r})\right]
$$

which has been regularized with the regularization functions $R_{m}(\varepsilon, \Lambda)$ defined in eq. (18) 21]. The axial density determines the axial-vector coupling constant of the proton

$$
g_{A}=-2 \int d^{3} r A_{o}(\boldsymbol{r})
$$

where an additional factor $(-1 / 3)$ is incorporated which results from the projection onto the isospin quantum number $T=1 / 2$ of the proton 23].

The total energy $E$ of a static quark-meson configuration is given by [16]

$$
E=E_{v a l}^{q}+E_{\text {sea }}^{q, R e g}+E^{M}+E^{C S B}
$$

with the valence-quark energy 


$$
\begin{aligned}
E_{\text {val }}^{q} & =\frac{1}{\mathcal{T}}\left[1-\mu \frac{\partial}{\partial \mu}\right] \mathcal{A}_{\text {val }}^{q}=\frac{N_{c}}{2} \sum_{\alpha}\left[\operatorname{sign}\left(\mu-\varepsilon_{\alpha}\right)+\operatorname{sign}\left(\varepsilon_{\alpha}\right)\right] \varepsilon_{\alpha} \\
& =N_{c} \theta\left(\varepsilon_{v a l}\right) \varepsilon_{v a l}
\end{aligned}
$$

and regularized sea-quark contributions

$$
E_{\text {sea }}^{q, \text { Reg }}=-\frac{N_{c}}{2} \sum_{\alpha}\left[R_{E}\left(\varepsilon_{\alpha}, \Lambda\right)\left|\varepsilon_{\alpha}\right|-R_{E}\left(\varepsilon_{\alpha}^{V}, \Lambda\right)\left|\varepsilon_{\alpha}^{V}\right|\right]
$$

The meson energy is given by

$$
E^{m}=m f_{\pi} \frac{\lambda^{2}}{g} 4 \pi \int r^{2} d r[1-\cos \Theta(r)] .
$$

For time-independent fields the classical meson and sea-quark energies differ from the corresponding effective actions (5, 9) only by a factor $\mathcal{T}$. An additional contribution to the energy results from $\mu$ dependence of the valence-quark action (7).

Finally we consider two spurious contributions to the quark energy which result from the mean-field approximation and have to be subtracted from the total energy (28). As shown in [5, 22] the static hedgehog contains a center-of-mass motion with the energy

$$
E_{C M M}=\frac{\left\langle: \boldsymbol{P}^{2}:\right\rangle}{2 E}=\frac{\left\langle: \int d^{3} r q^{\dagger}(\boldsymbol{r})\left(-\nabla^{2}\right) q(\boldsymbol{r}):\right\rangle}{2 E},
$$

where $\mathrm{E}$ is the rest mass represented by the total hedgehog energy (28). The expectation value of the square of the total quark momentum $\boldsymbol{P}$ consists of a valence- and a seacontribution given by

$$
\left\langle\boldsymbol{P}^{2}\right\rangle_{v a l}=N_{c} \theta\left(\varepsilon_{v a l}\right) \int d^{3} r \Phi_{v a l}^{\dagger}(\boldsymbol{r})\left(-\nabla^{2}\right) \Phi_{v a l}(\boldsymbol{r})
$$

and

$$
\begin{aligned}
&\left\langle\boldsymbol{P}^{2}\right\rangle_{\text {sea }}=-\frac{N_{c}}{2} \sum_{\alpha} {\left[R_{m}\left(\varepsilon_{\alpha}, \Lambda\right) \int d^{3} r \Phi_{\alpha}^{\dagger}(\boldsymbol{r})\left(-\nabla^{2}\right) \Phi_{\alpha}(\boldsymbol{r})-\right.} \\
&\left.R_{m}\left(\varepsilon_{\alpha}^{V}, \Lambda\right) \int d^{3} r \Phi_{\alpha}^{V \dagger}(\boldsymbol{r})\left(-\nabla^{2}\right) \Phi_{\alpha}^{V}(\boldsymbol{r})\right] .
\end{aligned}
$$

Another correction term results from the quark rotational degrees of freedom [23, 24, 25]. It is described by a moment of inertia $I$, which can be calculated within the semiclassical cranking approach [26] and consists of a valence contribution

$$
I_{v a l}=\frac{N_{c}}{2} \theta\left(\varepsilon_{v a l}\right) \sum_{\beta \neq v a l} \frac{\left\langle\Phi_{v} a l\left|\tau_{3}\right| \Phi_{\beta}\right\rangle\left\langle\Phi_{\beta}\left|\tau_{3}\right| \Phi_{v} a l\right\rangle}{\varepsilon_{\beta}-\varepsilon_{\alpha}}
$$

and of a regularized sea contribution

$$
I_{\text {sea }}=\frac{N_{c}}{2} \sum_{\alpha \beta} R_{I}\left(\varepsilon_{\alpha}, \varepsilon_{\beta} ; \Lambda\right) \frac{\left\langle\Phi_{\alpha}\left|\tau_{3}\right| \Phi_{\beta}\right\rangle\left\langle\Phi_{\beta}\left|\tau_{3}\right| \Phi_{\alpha}\right\rangle}{\varepsilon_{\beta}-\varepsilon_{\alpha}} .
$$

Within the proper-time scheme the regularization function is given by 


$$
\begin{aligned}
& R_{I}\left(\varepsilon_{\alpha}, \varepsilon_{\beta} ; \Lambda\right)= \\
& =\frac{1}{2} \frac{1}{\sqrt{4 \pi}} \int_{1 / \Lambda^{2}}^{\infty} d s s^{-\frac{3}{2}} \frac{1}{\varepsilon_{\beta}+\varepsilon_{\alpha}}\left[e^{-s \varepsilon_{\alpha}^{2}}-e^{-s \varepsilon_{\beta}^{2}}+s\left(\varepsilon_{\beta}-\varepsilon_{\alpha}\right)\left(\varepsilon_{\alpha} e^{-s \varepsilon_{\alpha}^{2}}+\varepsilon_{\beta} e^{-s \varepsilon_{\beta}^{2}}\right)\right] \\
& =\frac{1}{4}\left[\operatorname{sign}\left(\varepsilon_{\beta}\right) \operatorname{erfc}\left(\frac{\left|\varepsilon_{\beta}\right|}{\Lambda}\right)-\operatorname{sign}\left(\varepsilon_{\alpha}\right) \operatorname{erfc}\left(\frac{\left|\varepsilon_{\alpha}\right|}{\Lambda}\right)-\frac{2}{\sqrt{\pi}} \frac{e^{-\left(\frac{\varepsilon_{\alpha}}{\Lambda}\right)^{2}}-e^{-\left(\frac{\varepsilon_{\beta}}{\Lambda}\right)^{2}}}{\left(\varepsilon_{\alpha}+\varepsilon_{\beta}\right) / \Lambda}\right] .
\end{aligned}
$$

In the limit $\Lambda \rightarrow \infty$ one gets the well-known Inglis formula [27] for the moment of inertia. The incomplete error-function is given by $\operatorname{erfc}(x)=\frac{2}{\sqrt{\pi}} \int_{x}^{\infty} e^{-t^{2}} d t$. Since the energy correction for the nucleon and the $\Delta$ isobar are different the moment of inertia gives rise to a mass splitting between both particles which is given by [25]

$$
E_{\Delta}-E_{N}=\frac{3}{2 I}
$$

\section{The self-consistent meson profile and its depen- dence on the constituent quark mass}

The equation of motion (13) is an implicite and nonlocal equation for the profile function $\Theta(r)$. Because of the dependence of the expectation values $\bar{S}(r)$ and $\bar{P}(r)$ on the eigenvalues and eigenfunctions of the hamiltonian $h$ the right-hand side of the equation of motion is a functional of the profile function. We determine $\Theta(r)$ iteratively. Starting from a reasonable profile $\Theta^{o}(r)$ we determine eigenfunctions $\Phi_{\alpha}^{o}(\boldsymbol{r})$ and eigenvalues $\varepsilon_{\alpha}^{o}$ by diagonalizing the hamiltonian (14) within an appropriate basis. By means of the equation of motion (13) and the auxileary functions (14 17 ) we get an improved profile function $\Theta^{1}(r)$. Continuing this procedure to convergency we get the self-consistent profile function.

We represent the eigenfunctions of the hamiltonian within a discrete basis introduced in [28]. It is defined within a spherical box with a radius $D$ which is several times larger than the extension of $\Theta$ field. Details of the procedure are described in [10, 11, 16].

For all our calculation a box radius $D=15 / M$ has turned out to be sufficiently large. The discrete basis was limited by a maximal momentum $K_{\max }=8 \mathrm{M}$, which is more than 4 times larger than the corresponding regularization parameter $\Lambda$.

We have numerically determined the self-consistent profiles for 13 values of the constituent quark mass $M$ between 350 and $1000 \mathrm{MeV}$. As illustrated in fig. 1 the profiles are nearly independent of the mass parameter. None of the calculated profiles leaves the narrow corridor limited be the two broken lines. At masses lower than $350 \mathrm{MeV}$ the iteration converges to the vaccum field $(\Theta(r) \equiv 0)$. Here the corresponding interaction strength $G$ is not strong enough to keep a solitonic configuration together.

The self-consistent profiles can be approximated by a reference profile

$$
\Theta^{R e f}(R ; r)= \begin{cases}-\pi\left(1-\frac{r}{2 R}\right) & \text { if } r \leq R_{M} \\ -\pi\left(1-\frac{R_{M}}{2 R}\right)\left(\frac{R_{M}}{r}\right)^{2} \frac{1+m_{\pi} r}{1+m_{\pi} R_{M}} e^{-m_{\pi}\left(r-R_{M}\right)} & \text { if } r \geq R_{M}\end{cases}
$$

with the matching point 


$$
R_{M}=\frac{4}{3} R\left(1+\frac{8}{27} m_{\pi}^{2} R^{2}+\mathcal{O}\left[\left(m_{\pi} R\right)^{3}\right]\right)
$$

and an empirically determined radius parameter

$$
R=0.42 \mathrm{fm} .
$$

The reference profile (39) with the matching point (40) interpolates smoothly between the correct asymptotic behaviour at $r \rightarrow 0$ and $r \rightarrow \infty$ following from the asymptotic expansion of the equation of motion (13). It constitutes - at least visually - a fair approximation of the self-consistent profiles obtained after a time-consuming iteration procedure. Further tests of the reference profile will be performed in the next section.

\section{Testing the reference profile on nucleon observab- les}

In sect. 2 we considered several expectation values characterizing a quark or meson configuration. All these quantities are functionals of the profile function within our model. We have tested the quality of the reference profile (39) and evaluated the observables using both the reference and the self-consistently determined profiles. Fig. 2 shows the total energy (28) and its components (29, 30, 31), the mean square radii (23) of the baryon density, including their valence- and sea-quark contributions, and the axial-vector coupling constant of the proton (27) calculated with either profile. The kinks in the valence and sea contributions at the critical constituent mass $M=M_{d i p} \approx 750 \mathrm{MeV}$ result from the valence level which leaves the Fermi sea and joins the Dirac sea. The behaviour of the regularization functions (10, 18, 37) at $\varepsilon \rightarrow 0$ guarantees that the sum of valence and regularized sea contributions is a smooth function of the constituent quark mass $M$. Fig. 2 illustrates that nicely.

The only noticeable difference between the values for self-consistent and reference profiles appears in the valence and sea contributions in the vicinity of $M_{d i p}$. For the self-consistently determined profiles, the valence level dips into the Dirac sea at $M \approx$ $750 \mathrm{MeV}$. This point is shifted to $M \approx 725$ for the reference profile. The deviation is another evidence for the more sensitive dependence of valence and sea contributions on details of the profile function, while their sum is quite insensitive. One should note, however, that the physically relevant region for the constituent mass is around $M=$ $400 \mathrm{MeV}$, where the nucleon observables get reproduced by the reference profile very well.

The calculated nucleon observables are in sufficient agreement with similar calculations [16, 21, 17]. The too small value of the axial-vector coupling constant $\left(g_{A} \approx 0.6 \sim 0.8\right)$ in comparison to the experimental value $\left(g_{A}^{e x p} \approx 1.25\right)$ is a lack shared by many chiral models of the nucleon. However it is rather the aim of this paper to compare between two theoretical approaches than to reproduce the experimental values.

To complete our test of the reference profile we evaluate corrections to the static hedgehog configurations due to zero-point modes and spin-isospin quantization. Fig. 3 shows the energy $E_{C M M}$ (32) of the center-of-mass motion, the moment of inertia $I$ including the components (35) and (36), and the resulting nucleon-delta mass splitting (38). We establish an excellent agreement between the values for both kinds of profiles in the physically relevant mass region below $600 \mathrm{MeV}$. Larger deviations appear at $M \gtrsim$ 
$600 \mathrm{MeV}$. Here the energy corrections are so large that the perturbative approach used for their determination is already not justified.

\section{Conclusions}

We have self-consistently calculated average meson fields for the SU(2) Nambu \& JonaLasinio model with scalar-isoscalar and pseudoscalar-isovector couplings in Hartree approximation. The fields are restricted to the chiral circle and to hedgehog configurations. Infinite quark contributions are regularized within Schwinger's poper-time scheme.

The numerically determined meson profile functions turn out to be nearly independent of the constituent quark mass. They can be approximated quite well by a unique reference profile given by a simple arithmetic expression, which interpolates between the correct asymptotic behaviour at large and small radii. It is shown that the reference profile does not only approximate the self-consistent profiles but also reproduces the relevant observables of the quark and meson configurations.

We conclude that many of the properties of the Nambu \& Jona-Lasinio lagrangian can be studied using the reference profile instead of applying the time-consuming determination of the self-consistent profile. Changing the constituent quark mass $M$ only the strength $g$ of the quark-meson coupling is influenced $(g \sim M)$, while shape of the meson fields is almost independent of $M$. The absolute strength of the meson fields was fixed by the restriction to the chiral circle which can be justified from an extended NJL model implementing the trace anomaly of QCD [29, 30, 31]. If an accurate determination of the self-consistent profile is necessary, the reference profile may serve as a suitable starting profile.

The reference profile can be compared with the Woods-Saxon potential describing the average field inside an atomic nucleus. Most of the nuclear properties are sufficiently well described by this model potential which is rather determined by a simple ansatz (with 3 parameters) than by a sophisticated Hartree or Hartree-Fock procedure.

R. W. thanks for repeated hospitality of the University of Bochum. The work has partially been supported by the Bundesministerium für Forschung und Technologie, Bonn (contract 06 DR 107), the COSY-Projekt of the KFA Jülich the Alexander-von-Humboldt-Foundation (FeodeorLynen program) and by the Department of Energy (grant DE-FG06-90ER40561). 


\section{References}

[1] Nambu, J., Jona-Lasinio, G.: Phys. Rev. 122 (1961) 345; 124 (1961) 246

[2] Vogl, U., Weise, W.: Prog. Part. and Nucl. Phys. 27 (1991) 195

[3] Klevansky, S.: Rev. Mod. Phys. 64 (1992) 649

[4] Hatsuda, T., Kunihiro, T.: Phys. Rep. (1994) to be published

[5] Meißner, Th., Blotz, A., Ruiz Arriola, E., Goeke, K.: Rep. Prog. Phys. (1994) to be published

[6] Eguchi, T.: Phys. Rev. D14 (1976) 2755

[7] Kleinert, H.: 1976 Erice Summer Institute: Understanding the fundamental constituents of matter, Plenum Press, NY, (1978) (ed. by A. Zicchichi)

[8] Dyakonov, D., Petrov, V., Pobylitsa, P.: Nucl.Phys. B306 (1988) 809

[9] Meißner, Th., Ruiz Arriola, E., Grümmer, F., Goeke, K., Mavromatis, H.: Phys. Lett. B214 (1988) 312

[10] Reinhardt, H, Wünsch, R.: Phys. Lett. B215 (1988) 577; B230 (1989) 93

[11] Meißner, Th., Grümmer, F., Goeke, K.: Phys. Lett. B227 (1989) 296

[12] Dyakonov, D., Petrov, V., Pobylitsa, P. V., Praszalowicz, M.: Nucl. Phys. B323 (1989) 53

[13] Praszalowicz, M.: Phys. Rev. D42 (1990) 216

[14] R. Alkofer Phys. Lett. B236 (1990) 310

[15] Th. Meißner, F. Grümmer, K. Goeke Ann. Phys. 202 (1990) 297

[16] Th. Meißner and K. Goeke Nucl. Phys. A524 (1991) 719

[17] M. Wakamatsu and H. Yoshiki Nucl. Phys. A524 (1991) 561

[18] A. Chodos, B. Thorn Phys. Rev. D12 (1975) 2733

[19] D. Ebert, H. Reinhardt Nucl. Phys. B271 (1986) 188

[20] J. Schwinger Phys. Rev. 82 (1951) 664

[21] Th. Meißner, K. Goeke Z. Phys. A339 (1991) 513

[22] P. V. Pobylitsa, E. Ruiz Arriola, Th. Meißner, F. Grümmer, K. Goeke, W. Broniowski J. Phys. G18 (1992) 1455

[23] G. S. Adkins, C. R. Nappi, E. Witten Nucl. Phys. B228 (1983) 552

[24] T. D. Cohen, W. Broniowski Phys.Rev. D34 (1986) 3472

[25] H. Reinhardt Nucl.Phys. A503 (1989) 825 
[26] P. Ring and P. Schuck The nuclear many-body problem (Springer, New York, 1980)

[27] D. R. Inglis Phys. Rev. 96 (1954) 1059

[28] S. Kahana, G. Ripka Nucl. Phys. A419 (1984) 462

[29] G. Ripka and M. Jaminon Ann. Phys. (NY) 218 (1992) 51

[30] Th. Meißner, G. Ripka, R. Wünsch, P. Sieber, F. Grümmer and K. Goeke Phys. Lett. B299 (1993) 183

[31] C. Weiss, R. Alkhofer and H. Weigel Mod. Phys. Lett. A8 (1993) 79 


\section{FIGURE CAPTIONS}

1. Self-consistet profiles in the mass region $350 \mathrm{MeV} \leq M \leq 1000 \mathrm{MeV}$ All selfconsistently calculated profiles fit in the area marked by the broken lines.

The full line represents the reference profile (39) with $R=0.42 \mathrm{fm}$.

2. Nucleon observables in dependence on the constituent quark mass $M$ calculated with self-consistently determined profiles (full lines) in comparison to the reference profile $\Theta^{R e f}(R ; r)$ defined in eq. (39) with $R=0.42 \mathrm{fm}$. (broken lines)

Upper part: Total energy $E(28)$ and its components $E_{\text {val }}(29), E_{\text {sea }}^{\text {Reg }}(30)$ and $E^{m}$ (31).

Central part: mean square radius $\bar{R}(23)$ and its valence $\left(\bar{R}_{v a l}\right)$ and sea contributions $\left(\bar{R}_{\text {sea }}\right)$ calculated with the corresponding densities (21) and (22), respectively.

Lower part: Proton axial-vector coupling constant $g_{A}$ (27) (total value only).

3. Energy corrections to the static hedgehog energy calculated with self-consistently determined profiles (full lines) and with the reference profile (broken lines) in dependence on the constituent quark mass $M$.

Upper part: Energy of the center-of-mass motion $E_{C M M}$ (32).

Central part: Moment of inertia $I$ as the sum of the valence part $I_{v a l}$ (35) and the sea contributions $I_{\text {sea }}$ (36).

Lower part: Delta-nucleon mass splitting (38). 
This figure "fig1-1.png" is available in "png" format from: http://arxiv.org/ps/nucl-th/9311017v1 
This figure "fig1-2.png" is available in "png" format from: http://arxiv.org/ps/nucl-th/9311017v1 
This figure "fig1-3.png" is available in "png" format from: http://arxiv.org/ps/nucl-th/9311017v1 